OPEN ACCESS

Edited by: Yanghua Tian,

First Affiliated Hospital of Anhu Medical University, China

Reviewed by: Gaelle Eve Doucet,

Boys Town National Research Hospital, United States Takefumi Ueno, Hizen Psychiatric Center (NHO), Japan

*Correspondence:

Hui Zhong 313956777@qq.com

Specialty section

This article was submitted to Neuroimaging and Stimulation, a section of the journal

Frontiers in Psychiatry

Received: 19 October 2021 Accepted: 18 January 2022 Published: 10 February 2022

Citation:

Guo $P$, Hu S, Jiang $X$, Zheng $H, M o D$,

Cao X, Zhu J and Zhong H (2022) Associations of Neurocognition and Social Cognition With Brain Structure and Function in Early-Onset Schizophrenia.

Front. Psychiatry 13:798105. doi: 10.3389/fpsyt.2022.798105

\section{Associations of Neurocognition and Social Cognition With Brain Structure and Function in Early-Onset Schizophrenia}

\author{
Pengfei Guo ${ }^{1}$, Shuwen Hu${ }^{1}$, Xiaolu Jiang ${ }^{1}$, Hongyu Zheng ${ }^{1}$, Daming $\mathrm{Mo}^{2}$, Xiaomei Cao ${ }^{2}$, \\ Jiajia Zhu ${ }^{3}$ and Hui Zhong ${ }^{1,2 *}$ \\ ${ }^{1}$ Department of Child and Adolescent Mental Disorder, Affiliated Psychological Hospital of Anhui Medical University, Hefei, \\ China, ${ }^{2}$ Department of Child and Adolescent Mental Disorder, Anhui Mental Health Center, Hefei, China, ${ }^{3}$ Department of \\ Radiology, First Affiliated Hospital of Anhui Medical University, Hefei, China
}

Background: Cognitive impairment is a core feature of schizophrenia that is more serious in patients with early-onset schizophrenia (EOS). However, the neuroimaging basis of cognitive functions, including neurocognition and social cognition, remains unclear in patients with EOS.

Methods: Forty-three patients with EOS underwent structural and resting state functional magnetic resonance imaging scans. Brain structure and function were evaluated through the analysis of brain gray matter volume (GMV) and amplitude of low-frequency fluctuations (ALFF). They underwent comprehensive assessments for neurocognition (verbal memory, verbal expression, attention, and executive function) and social cognition (theory of mind and attributional bias). Correlation analyses were conducted to detect the potential link between cognitive function indices and brain imaging parameters.

Results: First, neurocognition was linked to brain structure characterized by higher immediate recall scores associated with increased GMV in the left temporal pole, higher verbal fluency scores associated with increased GMV in the left temporal pole: middle temporal gyrus, and higher Stroop-word scores associated with increased GMV in the right middle frontal gyrus. Second, social cognition was related to brain function characterized by lower sense of reality scores associated with increased ALFF in the left precentral gyrus, higher scores of accidental hostility bias associated with increased ALFF in the right middle temporal gyrus, and higher scores of accidental aggression bias associated with increased ALFF in the left precentral gyrus.

Conclusion: These findings may add to the existing knowledge about the cognitive function-brain relationship. They may have clinical significance for studying the mechanism of neurocognitive and social cognitive impairment in patients with EOS and providing potential neural targets for their treatment and intervention.

Keywords: early-onset schizophrenia, neurocognition, social cognition, gray matter volume, amplitude of lowfrequency fluctuation 


\section{INTRODUCTION}

Schizophrenia is a chronic and declining psychiatric disorder that affects nearly $1 \%$ of the world's population, and commonly occurs in late adolescence or early adulthood (1). Approximately $11 \%$ of patients had onset of schizophrenia before the age of 18 (2), which is defined as early-onset schizophrenia (EOS) (3). Compared with adult-onset schizophrenia, adolescents with EOS have significantly worse symptoms and social outcomes characterized by a chronic illness course, insidious onset, and long treatment delays $(4,5)$. Cognitive dysfunction is a core feature of schizophrenia and can predict functional outcomes $(6,7)$. Furthermore, after standard treatment with antipsychotic drugs, the cognitive function of patients with schizophrenia cannot be completely improved and may require adjuvant treatments $(8,9)$. The refractoriness of antipsychotics may be closely related to the early onset of psychotic symptoms. Data from a prospective trial have shown that EOS is more likely to be refractory than adult-onset schizophrenia and have more severe cognitive impairment (10). The results of a longitudinal study have indicated a neurodevelopmental pathway of EOS with subnormal cognitive development specific to adolescence (11). Therefore, a full understanding of the neurobiological basis of cognitive impairment in patients with EOS may have important clinical significance.

In the past, many efforts have been made to explore the mechanisms of cognitive impairment in schizophrenia. It has been reported that cognitive dysfunction in schizophrenia can be improved by stimulating the glycine regulatory site of the $\mathrm{N}$-methyl-d-aspartate-type glutamate receptor $(12,13)$. Fachim et al. further pointed out that changes in GRIN2B promoter methylation may lead to glutamatergic dysfunction in psychosis and are related to reduced cognitive performance in patients with first-episode schizophrenia (14). Data from electroencephalogram studies revealed that sleep spindle deficits in patients with schizophrenia can predict lower cognitive performance; this includes patients with EOS (15). A previous study suggested that sleep spindle deficits may be caused by dysfunction of the thalamic cortical network (16). A neuroimaging study using diffusion tensor imaging and tractography methods also found that lower fractional anisotropy of the left inferior fronto-occipital fasciculus and left inferior longitudinal fasciculus can predict worse neurocognitive performance

Abbreviations: AIHQ, Ambiguous Intentions Hostility Questionnaire; ALFF, amplitude of low-frequency fluctuation; AVLT, auditory verbal learning test; BOLD, blood-oxygen-level-dependent; DARTEL, diffeomorphic anatomical registration through the exponentiated lie algebra; DPABI, Data Processing \& Analysis for Brain Imaging; EOS, early onset schizophrenia; FA, flip angle; FD, frame-wise displacement; FOV, field of view; FWE, family wise error; GMV, gray matter volume; MFG, middle frontal gyrus; MNI, Montreal Neurological Institute; MRI, magnetic resonance imaging; MTG, middle temporal gyrus; $P r$, partial correlation coefficient; ROI, region of interest; rTPJ, right temporoparietal junction; SD, standard deviation; TE, echo time; TIV, total intracranial volume; TP, temporal pole; TR, repetition time; VBM, voxel-based morphometry; VFT, verbal fluency test. in EOS (17). Although these findings contribute to the understanding of the mechanism of cognitive impairment in EOS, the complex association between them still needs further elucidation.

A safe, non-invasive, and easily reproducible neuroimaging approach has been provided for in vivo human brain exploration through advances in magnetic resonance imaging (MRI) techniques $(18,19)$. Voxel-based morphometry (VBM) can be used to analyze the gray matter volume (GMV) in structural MRI (20). The amplitude of low-frequency fluctuation (ALFF) measures the low-frequency oscillation intensity of bloodoxygen-level-dependent (BOLD) time courses in resting-state functional MRI and reflects local neural activity strength (21). Using these methods, some studies have revealed changes in brain structure and function in patients with EOS (22-24). Nevertheless, there is a paucity of structural and functional MRI studies exploring the neural mechanisms of cognitive impairment in patients with EOS. Only a few related studies have reported that the pathophysiological mechanism of widespread cognitive impairment in EOS may be linked to abnormal changes in the GMV (25). For example, Kadriu et al. found that the development of early cognitive deficits in EOS may occur either concomitantly or closely with changes in the hippocampal volume (26). Shi et al. indicated that structural changes and disturbed resting-state functional connectivity in the core empathy network may correlate with the social cognitive deficits in patients with EOS (27). However, these previous studies have been largely limited to a portion of the cognitive functions of patients; they have paid less attention to the global cognitive functions, including neurocognition and social cognition.

A growing body of research showed that cognitive impairment in schizophrenia involves two dimensions: neurocognition and social cognition. Similar to adult-onset cases, EOS patients had neurocognitive and social cognitive impairments (28). Neurocognition is the basic function of the central nervous system, which includes attention and working memory, verbal memory, executive function, thought disorder, and processing speed. Social cognition refers to the ability to recognize and explain the emotions or intentions of others, and the ability to use these social signals to guide conclusions or behaviors, including theory of mind, attribution bias, emotional processing, social knowledge, and social perception (29-32). These dimensions are different but highly correlated (33). Bell et al. suggested that neurocognition leads to different rehabilitation outcomes by affecting social cognition (30).

In this study, neuropsychological tools were used to evaluate the neurocognitive and social cognitive functions of patients with EOS. These included verbal memory and expression, attention and executive function, theory of mind, and attribution bias. VBM and ALFF analyses were used to measure brain structure and function. The associations between global cognitive functions and brain regions in patients with EOS at the level of brain GMV and local neural activity were explored through correlation analyses. 


\section{MATERIALS AND METHODS}

\section{Participants}

This study consisted of 43 Chinese Han, right-handed patients with EOS within a restricted age range of 13-18 years. All patients were recruited consecutively from the Department of Child and Adolescent Mental Disorder, Affiliated Psychological Hospital of Anhui Medical University, Hefei. According to the International Classification of Diseases criteria, two welltrained clinical psychiatrists confirmed patients' diagnosis of EOS. The study's exclusion criteria were as follows: (1) the presence of other psychiatric disorders such as intellectual disability, bipolar disorder, substance-induced mood disorder, anxiety disorder, substance abuse or dependence; (2) a history of significant neurological or physical diseases; and (3) pregnancy or any contraindications for MRI. After a training to ensure consistent methodology of rating, two investigators completed the questionnaire assessment of patients. This study was approved by the Ethics Committee of the Affiliated Psychological Hospital of Anhui Medical University. After a complete description of the study was provided, all participants' legal guardians gave written informed consent. The demographic data of the participants are listed in Table $\mathbf{1}$ and behavioral outcomes of the cognitive tests are listed in Supplementary Table 1.

\section{Cognition Assessment} Neurocognition Assessment

The auditory verbal learning test (AVLT) was performed to evaluate verbal memory function, which included four indices such as immediate recall, short-term delayed recall, long-term delayed recall, and long-term delayed recognition (34). The test contains two different learning materials, each with 15 common Chinese vocabulary items. Participants would score one point for each correct recall of a word. In the immediate recall test, participants listened to the first set of words five times and recalled the words they heard within 2 min after each listening. Investigators recorded the score each time and calculated the average score as "immediate recall." Then, participants listened to the second set of words and immediately recalled it within 2 min after listening. After that, participants were asked to recall the

TABLE 1 | Demographic characteristics.

\begin{tabular}{lll}
\hline Characteristics & Mean \pm SD & Range \\
\hline Sex (female/male) & $15 / 28$ & - \\
Age (years) & $16.63 \pm 1.36$ & $13-18$ \\
Education (years) & $10.37 \pm 1.51$ & $7-12$ \\
FD (mm) & $0.13 \pm 0.07$ & $0.04-0.35$ \\
TIV $\left(\mathrm{cm}^{3}\right)$ & $1401.54 \pm 108.84$ & $1186.71-1629.88$ \\
Chlorpromazine equivalent doses & $389.21 \pm 99.93$ & $115.74-528.17$ \\
Course of disease (months) & $18.77 \pm 14.92$ & $2-51$ \\
The age of onset of schizophrenia & $15.07 \pm 4.56$ & $12-18$ \\
(years) & & \\
\hline
\end{tabular}

$S D$, standard deviation; FD, frame-wise displacement; TIV, total intracranial volume. first set of words, and investigators recorded the scores as "shortterm delayed recall." Thirty minutes after the fifth listening session, participants needed to recall the first set of words again, and the scores were noted as the "long-term delayed recall." Finally, participants were asked to listen to a 50-word vocabulary consisting of the first set of words, the second set of words and other interference words, and recognized the words in the first set. The score recognized successfully was used as an index of "long-term delayed recognition."

The verbal fluency test (VFT) was used to assess verbal expression (35). Each participant had to list as many names as possible of household appliances, animals, and fruits within $1 \mathrm{~min}$, respectively. Participants would score one point for each name. The investigators recorded the total scores as an index of verbal expression performance.

Attention was evaluated using the digit span tasks (36). The tasks consisted of two indices such as digit span forward and digit span backward. Span is defined as the maximum number of digits repeated by the participant. All participants completed a digit span forward task followed by a digit-span backward task. The former begins with a series of two digits orally presented to each participant, continuing to a maximum of 13 digits. Participants were asked to repeat the digits verbally. There were two trials per digit series. All participants began with the first digit series (i.e., two digits). If repeated correctly, the participant continued to the next one; otherwise, the second trial was performed with the same digit series. The task was discontinued when the participant failed in the second trial. The digit span backward task followed the same procedure, except that participants verbally repeated the digits in reverse order.

The Stroop color word test reflects executive function (37). There were three indices including Stroop-dot, Stroop-word and Stroop-color word in the test. The test materials consisted of three types of cards: A (dot), B (word), and C (color word). Card A had red, green, yellow, and blue dots arranged in a certain order in a $4 \times 4$ manner, and card $\mathrm{B}$ had four Chinese words (namely "Yin," “Zou," "Wen," "Sheng") with four colors of red, green, yellow, and blue arranged in the same order. Card C had four Chinese words (namely "red," "green," "yellow," "blue") with four colors of red, green, yellow, and blue arranged in the order of card A. The participants were asked to quickly read the color of the dots or Chinese words on the cards. The time taken to read each card was used as an index of executive function performance.

\section{Social Cognition Assessment}

The Theory of Mind Picture-Sequencing Task was performed to assess the ability of mentalization (38) through six stories. Each story consisted of four pictures, accompanied by $2 \sim 6$ questions. Participants composed a story with four pictures in a certain logical order and then answered questions from the investigators. Participants would get two points, respectively for the first and last pictures sequenced correctly of each story and get one point, respectively for the two pictures in the middle of each story. At the same time, one point would be awarded for each correct answer. These questions assessed the participants' ability to understand the mental state of the characters in the story, including primary beliefs, primary false beliefs, secondary 
beliefs, secondary false beliefs, tertiary false beliefs, sense of reality, understanding reciprocity, understanding deception, and detecting deception. Adding the total score, there were ten indices extracted from the test. The maximum score was 59. The scores were used as indices of mentalizing ability.

The Ambiguous Intentions Hostility Questionnaire (AIHQ) was used to evaluate attribution bias (39). The Chinese version of AIHQ is composed of three types of hypothetical scenarios describing the characters' behaviors: intentional (five vignettes), ambiguous (five vignettes), or accidental (five vignettes). After reading each vignette, participants were asked to imagine the scenario happening to them (e.g., "You walk past a bunch of teenagers at a mall and you hear them start to laugh"), and to write down why the other person (or persons) treated you this way. Two independent investigators subsequently rated this written response for the purpose of computing a "hostility index." Then, the participants rated, on Likert scales, whether the other person (or persons) performed the action on purpose ( 1 "definitely no" to 6 "definitely yes"), how angry it would make them feel (1 "not at all angry" to 5 "very angry"), and how much they would blame the other person (or persons) (1 "not at all" to 5 "very much"). Investigators calculated the average score of these three items as the "blame index." Finally, the participants were asked to write down how they would respond to the situation, which was later rated by two independent investigators to compute an "aggression index." For the hostility and aggression indices, two investigators independently evaluated each participant's reaction on 5-point Likert scales based on examples of high and low scores. The scales for the hostility and aggression indices were from 1 ("not at all hostile") to 5 ("very hostile"), and 1 ("not at all aggressive") to 5 ("very aggressive"), respectively. The hostility bias, blame bias, and aggression bias scores for each scenario type were averaged after being added up, respectively. Furthermore, the total scores for each bias in the three types of scenarios were added up as the total scores of hostility bias, total scores of blame bias, and total scores of aggression bias. On the whole, there were 12 indices extracted from the test. Higher scores indicated a more negative attribution bias.

\section{MRI Data Acquisition}

MRI scans were obtained using a 3.0-Tesla MR system (Discovery MR750w, General Electric, Milwaukee, WI, USA) with a 24channel head coil. Earplugs were used to reduce scanner noise, and tight but comfortable foam padding was used to minimize head motion. Before the scanning, all participants were instructed to keep their eyes closed, think of nothing in particular, move as little as possible, and relax but not fall asleep during the scans. High-resolution 3D T1-weighted structural images were acquired by employing a brain volume (BRAVO) sequence with the following parameters: repetition time $(\mathrm{TR})=8.5 \mathrm{~ms}$; echo time $(\mathrm{TE})=3.2 \mathrm{~ms}$; inversion time $=450 \mathrm{~ms}$; flip angle $(\mathrm{FA})=$ $12^{\circ}$; field of view $(\mathrm{FOV})=256 \mathrm{~mm} \times 256 \mathrm{~mm}$; matrix size $=$ $256 \times 256$; slice thickness $=1 \mathrm{~mm}$, no gap; voxel size $=1 \mathrm{~mm}$ $\times 1 \mathrm{~mm} \times 1 \mathrm{~mm} ; 188$ sagittal slices. Resting-state BOLD fMRI data were acquired using a gradient-echo single-shot echo planar imaging (GRE-SS-EPI) sequence with the following parameters:
$\mathrm{TR}=2,000 \mathrm{~ms} ; \mathrm{TE}=30 \mathrm{~ms} ; \mathrm{FA}=90^{\circ} ; \mathrm{FOV}=220 \mathrm{~mm} \times$ $220 \mathrm{~mm}$; matrix size $=64 \times 64$; slice thickness $=3 \mathrm{~mm}$, slice gap $=1 \mathrm{~mm} ; 35$ interleaved axial slices; voxel size $=3 \mathrm{~mm} \times$ $3 \mathrm{~mm} \times 3 \mathrm{~mm} ; 185$ volumes. After the scanning, all images were visually inspected to ensure that only images without visible artifacts, lesions, and regional deformations were included in subsequent analyses.

\section{Gray Matter Volume Analysis}

The 3D T1-weighted structural images were processed using the VBM8 toolbox (http://dbm.neuro.uni-jena.de/vbm.html) implemented in the Statistical Parametric Mapping software (SPM8, http://www.fil.ion. ucl.ac.uk/spm). First, all the structural images were segmented into gray matter, white matter, and cerebrospinal fluid density maps using the standard segmentation model. After an initial affine registration of the gray matter density map into the Montreal Neurological Institute (MNI) space, the gray matter density images were non-linearly warped using the diffeomorphic anatomical registration through the exponentiated lie algebra (DARTEL) technique (40). They were then resampled to a voxel size of $1.5 \mathrm{~mm} \times 1.5 \mathrm{~mm} \times$ $1.5 \mathrm{~mm}$. The GMV map was obtained by multiplying the gray matter density map by the non-linear determinants derived from the spatial normalization step. Finally, the resultant GMV images were smoothed with a Gaussian kernel of $8 \mathrm{~mm} \times 8 \mathrm{~mm} \times 8 \mathrm{~mm}$ full-width at half maximum.

\section{ALFF Analysis}

Resting-state BOLD data were preprocessed using SPM12 (http:// www.fil.ion.ucl.ac.uk/spm) and Data Processing \& Analysis for Brain Imaging (DPABI, http://rfmri.org/ dpabi) (41). The first ten volumes for each participant were discarded to allow the signal to reach equilibrium and the participants to adapt to the scanning noise. The remaining volumes were corrected for the acquisition time delay between slices. Then, realignment was performed to correct the motion between the time points. Head motion parameters were computed by estimating the translation in each direction and the angular rotation on each axis for each volume. All participants' BOLD data were within the defined motion thresholds (i.e., translational or rotational motion parameters $<2 \mathrm{~mm}$ or $2^{\circ}$, respectively). The frame-wise displacement (FD), which indexes the volume-to-volume changes in the head position, was also calculated. Several nuisance covariates (the linear drift, estimated motion parameters based on the Friston24 model, spike volumes with FD $>0.5$, white matter signal, and cerebrospinal fluid signal) were regressed out from the data. In the normalization step, individual structural images were first co-registered with the mean functional image; thereafter, the transformed structural images were segmented and normalized to the MNI space using the diffeomorphic anatomical registration through the exponentiated lie algebra (DARTEL) technique (40). Finally, each functional volume was spatially normalized to the MNI space using the deformation parameters estimated during the above step and resampled into a $3 \mathrm{~mm}$ cubic voxel. After spatial normalization, all data sets were smoothed with a $6 \mathrm{~mm}$ full-width at half maximum Gaussian kernel. 
ALFF analysis was conducted using DPABI software (http:// rfmri.org/ dpabi). After preprocessing, each voxel's BOLD time course was filtered (bandpass, $0.01-0.1 \mathrm{~Hz}$ ) to remove the effects of very-low-frequency drift and high frequency noise, e.g., respiratory and heart rhythms. The fast Fourier transform with default parameters from DPABI was used to transform the filtered BOLD time course of each voxel into frequency domain and the power spectrum was then obtained. Because the power of a given frequency is proportional to the square of the amplitude of this frequency component of the original BOLD time course in the time domain, the square root was calculated at each frequency of the power spectrum at each voxel. The averaged squared root was obtained across $0.01-0.1 \mathrm{~Hz}$, which was defined as the ALFF value. Finally, the ALFF value of each voxel was divided by the global mean ALFF value for standardization.

\section{Statistical Analysis}

The statistical descriptive analyses of demographic and behavioral data were conducted using the SPSS software (version 23.0; SPSS, Chicago, IL, USA). This study examined the relationship between cognitive function indices and brain imaging parameters in a voxel-wise manner within the whole gray matter in EOS patients. The general linear model in Statistical Parametric Mapping software (SPM12, http://www. fil.ion.ucl.ac.uk/spm) with a multiple regression design was used to identify any voxels in the GMV and ALFF maps that showed a significant association with the behavioral outcomes while controlling for age, sex, and years of education. Total intracranial volume (TIV) and FD were additional covariates for the GMV and ALFF analyses, respectively. The resulting maps were thresholded at an uncorrected voxelwise level of $p<$ 0.001 , and then considered significant at $p<0.05$ cluster-level family wise error (FWE)-corrected for multiple comparisons. For each subject, the GMV and ALFF values of each cluster with a significant correlation with behavioral outcomes were extracted by the RESTplus software (http://www.restfmri.net), and then used for region of interest (ROI)-based analyses that refers to the partial correlation analyses performed in SPSS while controlling the above covariates (Supplementary Tables 2, 3). To ensure the robustness of the findings, this study repeated the ROI-based partial correlation analyses after removing the outliers of outcomes (GMA and ALFF values, and behavioral outcomes) greater than mean $+3 \times$ standard deviation (SD) or smaller than mean $-3 \times \mathrm{SD}$. No corrections for multiple testing were conducted, as the objective for this study was to generate some hypotheses for further testing and confirmation in a larger sample.

\section{Sensitivity Analysis}

First, a previous study revealed the effect of antipsychotic drugs on brain structure and function (42). To test the possible effect of antipsychotic drugs on our results, chlorpromazine equivalent doses for antipsychotics were included as an additional nuisance covariate with repetition in the ROI-based partial correlation analyses (43). Second, the age of onset of schizophrenia might affect GMV and ALFF, so the ROI-based partial correlation analyses were repeated while adding this variable as an additional nuisance covariate.

\section{RESULTS}

\section{Correlations Between Neurocognition and GMV}

In the voxel-wise whole gray matter analysis, the significant correlations between neurocognition and GMV are shown in Figure 1 (cluster-level $P<0.05$, FWE corrected). After adjustment for age, sex, years of education, TIV, and outliers, significant positive correlations were found between the AVLTimmediate recall and GMV in the left temporal pole (TP) (cluster size: 1,602 voxels, peak MNI coordinate $\mathrm{x} / \mathrm{y} / \mathrm{z}$ : $-39 / 4.5 /-30$, peak $T$ : 4.872, partial correlation coefficient $[p r]: 0.626, P<0.001)$; positive correlations between the VFT and GMV in the left TP: middle temporal gyrus (cluster size: 762 voxels, peak MNI coordinate $\mathrm{x} / \mathrm{y} / \mathrm{z}$ : $-46.5 / 12 /-30$, peak $T: 5.319$, pr: $0.635, P<$ 0.001 ); and positive correlations between the Stroop-word and GMV in the right middle frontal gyrus (MFG) (cluster size: 1,289 voxels, peak MNI coordinate x/y/z: 40.5/22.5/42, peak $T: 4.951$, pr: $0.472, P=0.003)$. No significant correlations were observed between neurocognition and ALFF.

\section{Correlations Between Social Cognition and ALFF}

In the ALFF analysis, the significant correlations between social cognition and ALFF are shown in Figure 2 (cluster-level $P<$ 0.05, FWE corrected). After accounting for age, sex, years of education, FD, and outliers, the Theory of Mind-sense of reality showed significant negative correlations with ALFF in the left precentral gyrus (cluster size: 67 voxels, peak MNI coordinate x/y/z: $-21 /-24 / 66$, peak $T:-5.973$, pr: $-0.433, P=0.007)$. The accidental hostility bias exhibited significant positive correlations with ALFF in the right middle temporal gyrus (MTG) (cluster size: 51 voxels, peak MNI coordinate $x / y / z$ : 54/-36/-9, peak $T$ : 6.187, pr: $0.380, P=0.022$ ). There were significant positive correlations between the accidental aggression bias and ALFF in the left precentral gyrus (cluster size: 51 voxels, peak MNI coordinate $\mathrm{x} / \mathrm{y} / \mathrm{z}$ : $-30 /-24 / 45$, peak $T: 5.854$, pr: $0.377, P=$ $0.021)$. No significant correlations were observed between social cognition and GMV.

\section{Sensitivity Analysis}

First, after considering the influence of antipsychotics, the correlations between cognitive function indices and brain imaging parameters had slight changes in $\mathrm{p}$ values, but the main brain regions in the results had survived (Supplementary Tables 4, 5). Second, the correlations between cognitive function indices and brain imaging parameters remained unchanged after additionally adjusting for the age of onset of schizophrenia (Supplementary Tables 6, 7). These suggest that antipsychotics and the age of onset of schizophrenia did not influence the main results of this study. 
A

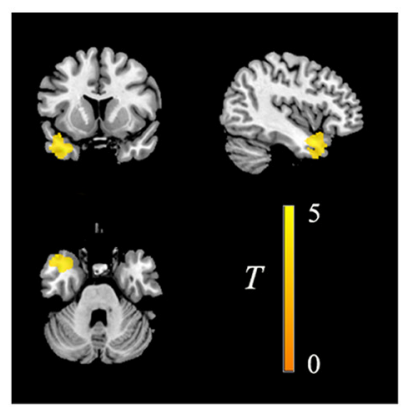

B

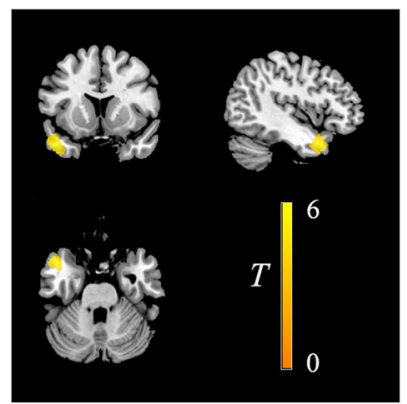

C

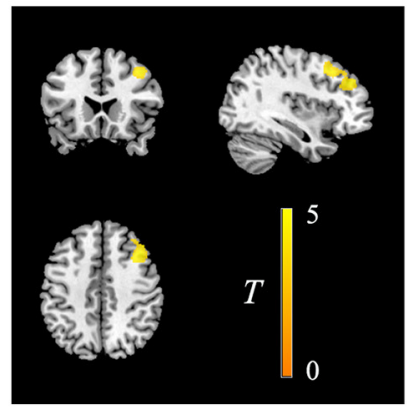

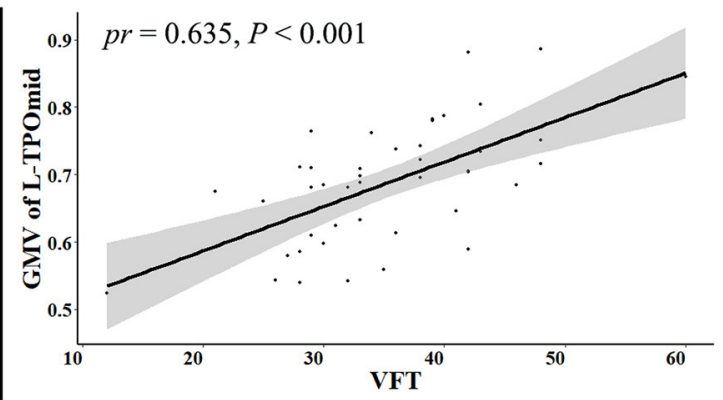

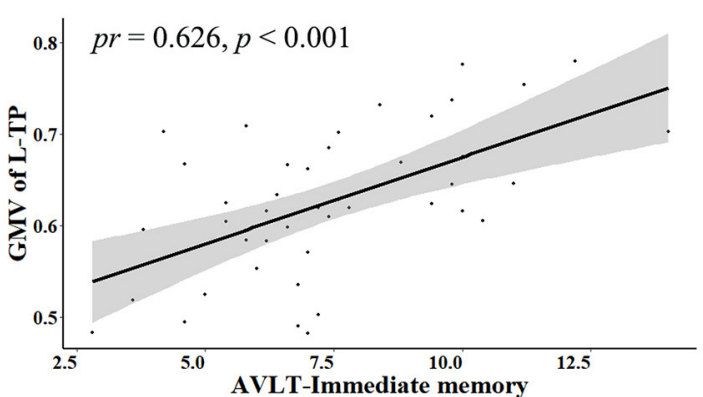

LT-Immediate memory

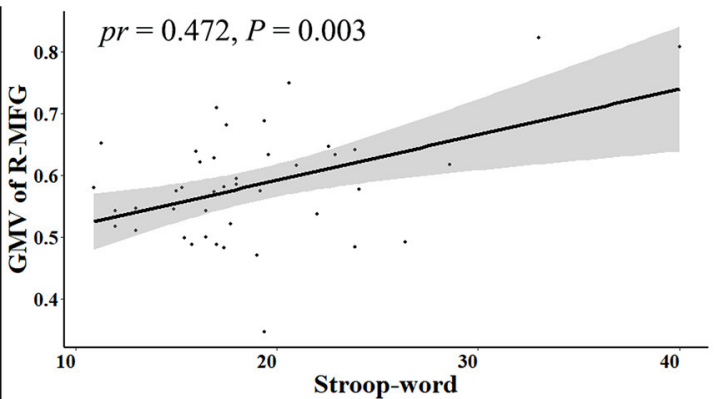

FIGURE 1 | Correlation between neurocognition and GMV. The left figure in (A-C) is voxel-based and the right is ROI-based. (A) Correlation between GMV in the left TP and AVLT-immediate recall. (B) Correlation between GMV in the left TPOmid and VFT. (C) Correlation between GMV in the right MFG and stroop-word. GMV, gray matter volume; ROI, region of interest; TP, temporal pole; AVLT, auditory verbal learning test; TPOmid, temporal pole: middle temporal gyrus; VFT, verbal fluency test; MFG, middle frontal gyrus; $p r$, partial correlation coefficient; L, left; R, right.

\section{DISCUSSION}

Using structural and resting-state functional MRI, we explored the relationships between neurocognitive function, social cognitive function, and brain imaging parameters in patients with EOS. There were two main findings in this study. The first was the relationship between neurocognitive function and brain structure. Higher language functions of verbal memory and expression were associated with increased GMV in the TP. Meanwhile, higher executive function was associated with increased GMV in the MFG. The second was the relationship between social cognitive function and brain function. This was characterized by lower theory of mind ability associated with increased ALFF in the precentral gyrus and more negative attribution bias with increased ALFF in the precentral gyrus, and MTG.
Previous studies have used VBM and ALFF methods to investigate changes in GMV and local neural activity in patients with schizophrenia (44-48). The TP, MFG, and MTG are the common brain areas consistently reported in previous studies and the current study. For instance, compared with healthy participants, some studies found that patients with schizophrenia demonstrate decreased GMV in the MFG and paralimbic system (including the orbitofrontal cortex, insular cortex, and TP) (44, 45). Li et al. found differences in the variability of dynamic ALFF between EOS patients and healthy controls in the bilateral precuneus, right superior marginal gyrus, right postcentral gyrus, and right MTG (48). However, the investigators focused solely on distinguishing this disease and paid no attention to changes in the global cognitive function of patients. We speculate that not only do EOS patients have altered GMV or local nerve activity in the common regions, but these alterations may also 
A
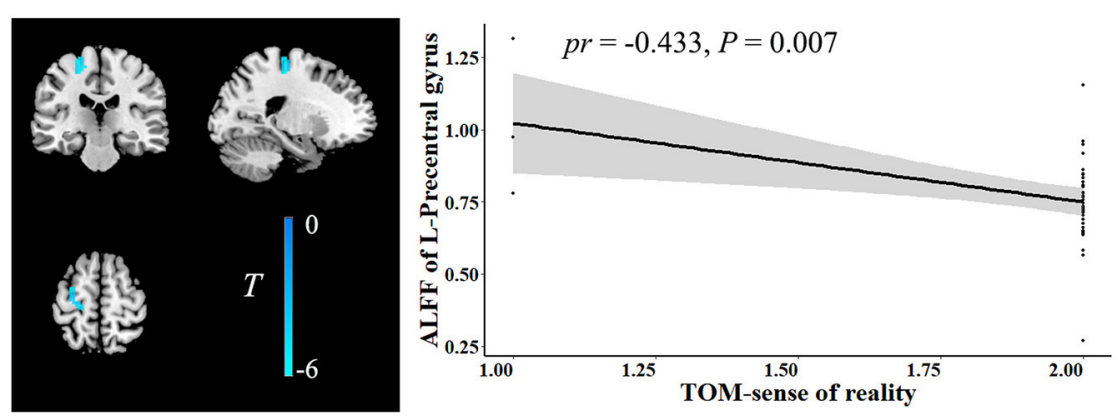

B
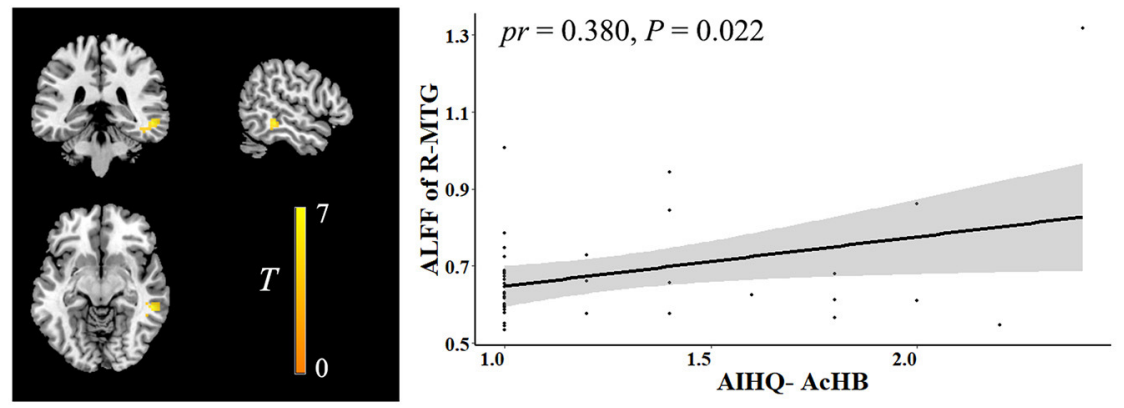

C
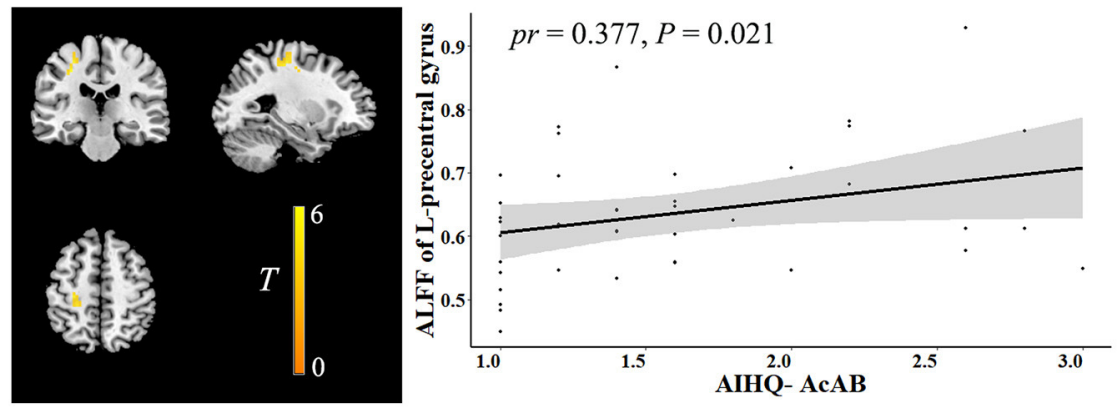

FIGURE 2 | Correlation between social cognition and ALFF. The left figure in (A-C) is voxel-based and the right is ROI-based. (A) Correlation between ALFF in the left precentral gyrus and TOM-sense of reality. (B) Correlation between ALFF in the right MTG and AlHQ-AcHB. (C) Correlation between ALFF in the left precentral gyrus and AlHQ-AcAB. ALFF, amplitude of low-frequency fluctuation; ROI, region of interest; TOM, theory of mind; AlHQ, ambiguous intentions hostility questionnaire; AcHB, accidental hostility bias; MTG, middle temporal gyrus; AcAB, accidental aggression bias; pr, partial correlation coefficient; L, left; R, right.

affect neurocognitive and social cognitive functions. There are some inconsistent findings between the current and previous studies. The differences across studies may be linked to patient characteristics (subtype of schizophrenia, age of onset, and course of disease), brain imaging methods (GMV, ALFF, and dynamic ALFF), and sample sizes.

The TP is the most rostral part of the temporal lobe, and its complex structure has been associated with a variety of advanced cognitive functions, including autobiographic memory (49-51), semantic memory $(52,53)$, generation of false memories (54), face processing (55) and processing of emotional understanding $(56,57)$. The "semantic hub" theory is one important theory about TP function, which considers the TP as a domaingeneral hub integrating semantic information from different modalities into a coherent representation $(58,59)$. Pehrs et al. showed that the TP plays a role in the integration of various semantic information and top-down modulation in the ventral visual stream (57). In patients with Alzheimer's disease, impaired semantic performance was associated with decreased GMV in the TP (60). Similarly, impaired semantic memory was also found in patients with epileptogenic lesions at the left TP (61). Consistent with these prior findings, a correlation between the TP and language function in EOS patients was found in this study. This suggests that damage to the TP may be a shared underlying neural mechanism leading to language dysfunction in a variety of neuropsychiatric diseases. The MFG is considered to be a component of the central executive network and involves processing of working memory information and the judgment and decision of goal-oriented behaviors (62-64). There is neuropsychological evidence that executive functions of schizophrenia, such as working memory and planning, are impaired (65), as well as neuroimaging evidence that specific deficits in an executive function known as goal maintenance in patients with schizophrenia are associated with reduced MFG 
activity (66). Collectively, these prior findings, coupled with the results of this study, suggest that MFG dysfunction may serve as the basis of executive function deficits in patients with schizophrenia, including EOS.

One interesting aspect of the results of this study was the negative correlation between social cognitive performance and local neural activity in the precentral gyrus and MTG in patients with EOS. The precentral gyrus is considered to be the core area of the mirror-neuron system and plays a fundamental role in both action understanding and imitation (67), associated with the realization of a variety of social cognitive functions $(68,69)$. Previous studies found that the aberrant connectivity of the mirror neuron system network may be linked to social dysfunction in patients with schizophrenia, and this aberrant connectivity might even exist in the early stages of psychosis $(70,71)$. Together with our data, it is reasonable to assume that functional deficits of the precentral gyrus may be a neural characteristic of the impaired social cognition in EOS patients. The MTG plays a vital role in social cognition, semantic processing, auditory processing, action observation and language (72). Increasing number of studies have reported that the MTG is also an important component of the Theory of Mind network. For example, Schurz et al. found significant activation in the MTG during the tasks of social animations, mind in the eyes, and rational actions (73). Another study reported that, compared with false beliefs, social animations showed the strongest activation in the MTG (74). In patients with schizophrenia, a diffusion-weighted MRI study reported an increased trace in the right MTG and a correlation between trace and decreased social cognition (75). Taken together, our results support the idea that MTG dysfunction serves as the substrate underlying impaired social cognition in the EOS.

There are some limitations that should be noted in this study. First, healthy controls were not included in this study. Although our results are similar to those of previous controlled studies with healthy controls, future investigations are warranted to further strengthen our findings more rigorously and accurately by enrolling a sample of subjects with EOS and healthy adolescents. Second, causal relationships cannot be inferred from this cross-sectional design. Longitudinal studies with interventions targeting the improvement of cognitive function in EOS are required to establish the direction of causality. Third, the patients were receiving antipsychotic medication that may have influenced the interpretation of results. Although the main results remained after adjusting for antipsychotic dose equivalents, the medication effect could not be completely eliminated (43). Future studies with medication-naïve firstepisode patients with EOS are needed to validate the preliminary findings of this study. Fourth, other indices (e.g., processing speed and emotional processing) would need to be analyzed to further clarify the cognitive function-brain relationship in the EOS. Finally, the results of this study were not corrected for multiple comparisons, because the results might not survive correction, likely due to the small sample size. However, our analyses were important for future hypothesis generation. Future studies are required to further expand the sample size and conduct the correction for multiple comparisons to make the results more reliable.

In conclusion, the present study is the first to explore the association between global cognitive function and the brain in patients with EOS. The observed relationships of neurocognition with GMV and social cognition with ALFF may help to expand the existing knowledge about the cognitive functionbrain relationship in EOS. To some extent, this disassociation between anatomy and function also supports that neurocognition is the basic cognitive function that affects the ability of daily living, and social cognition, which is a more advanced cognitive function, can better predict social outcome (29). More broadly, these findings may have clinical significance for studying the neurological mechanism of cognitive impairment in patients with EOS and provide potential neural targets for their treatment and intervention.

\section{DATA AVAILABILITY STATEMENT}

The original contributions presented in the study are included in the article/Supplementary Material, further inquiries can be directed to the corresponding author/s.

\section{ETHICS STATEMENT}

The studies involving human participants were reviewed and approved by the Ethics Committee of the Affiliated Psychological Hospital of Anhui Medical University. Written informed consent to participate in this study was provided by the participants' legal guardian/next of kin.

\section{AUTHOR CONTRIBUTIONS}

PG and SH: methodology, data curation, software, and writing original draft. XJ, HoZ, DM, and XC: data collection, visualization, and investigation. JZ: conceptualization, methodology, software, and formal analysis. $\mathrm{HuZ}$ : conceptualization, supervision, and writing-review and editing. All authors contributed to the article and approved the submitted version.

\section{FUNDING}

This work was supported by the grants of Anhui Provincial Department of Science and Technology (No. 1804h08020251) and National Key Research and Development Program (No. 2018YFC1314300).

\section{ACKNOWLEDGMENTS}

The authors give special thanks to the patients and their families.

\section{SUPPLEMENTARY MATERIAL}

The Supplementary Material for this article can be found online at: https://www.frontiersin.org/articles/10.3389/fpsyt. 2022.798105/full\#supplementary-material 


\section{REFERENCES}

1. Asarnow JR, Tompson MC, McGrath EP. Annotation: childhood-onset schizophrenia: clinical and treatment issues. J Child Psychol Psychiatry. (2004) 45:180-94. doi: 10.1111/j.1469-7610.2004.00213.x

2. Amminger GP, Henry LP, Harrigan SM, Harris MG, AlvarezJimenez M, Herrman $\mathrm{H}$, et al. Outcome in early-onset schizophrenia revisited: findings from the Early Psychosis Prevention and Intervention Centre long-term follow-up study. Schizophr Res. (2011) 131:112-9. doi: 10.1016/j.schres.2011.06.009

3. Werry JS. Child and adolescent (early onset) schizophrenia: a review in light of DSM-III-R. J Autism Dev Disord. (1992) 22:601-24. doi: 10.1007/ BF01046330

4. Joa I, Johannessen JO, Langeveld J, Friis S, Melle I, Opjordsmoen S, et al. Baseline profiles of adolescent vs. adult-onset first-episode psychosis in an early detection program. Acta Psychiatr Scand. (2009) 119:494500. doi: 10.1111/j.1600-0447.2008.01338.x

5. Hollis C. Adult outcomes of child- and adolescent-onset schizophrenia: diagnostic stability and predictive validity. Am J Psychiatry. (2000) 157:16529. doi: 10.1176/appi.ajp.157.10.1652

6. Reichenberg A, Harvey PD. Neuropsychological impairments in schizophrenia: integration of performance-based and brain imaging findings. Psychol Bull. (2007) 133:833-58. doi: 10.1037/0033-2909.133. 5.833

7. Harvey PD, Howanitz E, Parrella M, White L, Davidson M, Mohs RC, et al. Symptoms, cognitive functioning, and adaptive skills in geriatric patients with lifelong schizophrenia: a comparison across treatment sites. Am J Psychiatry. (1998) 155:1080-6. doi: 10.1176/ajp.155. 8.1080

8. Keefe RS, Bilder RM, Davis SM, Harvey PD, Palmer BW, Gold JM, et al. Neurocognitive effects of antipsychotic medications in patients with chronic schizophrenia in the CATIE Trial. Arch Gen Psychiatry. (2007) 64:633-47. doi: 10.1001/archpsyc.64. 6.633

9. Swartz MS, Perkins DO, Stroup TS, Davis SM, Capuano G, Rosenheck RA, et al. Effects of antipsychotic medications on psychosocial functioning in patients with chronic schizophrenia: findings from the NIMH CATIE study. Am J Psychiatry. (2007) 164:428-36. doi: 10.1176/ajp.2007.164. 3.428

10. Iasevoli F, Razzino E, Altavilla B, Avagliano C, Barone A, Ciccarelli M, et al. Relationships between early age at onset of psychotic symptoms and treatment resistant schizophrenia. Early Interv Psychiatry. (2021). doi: 10.1111/eip.13174. [Epub ahead of print].

11. Oie MG, Sundet K, Haug E, Zeiner P, Klungsoyr O, Rund BR. Cognitive performance in early-onset schizophrenia and attentiondeficit/hyperactivity disorder: a 25-year follow-up study. Front Psychol. (2020) 11:606365. doi: 10.3389/fpsyg.2020.606365

12. Javitt DC. Glutamate as a therapeutic target in psychiatric disorders. Mol Psychiatry. (2004) 9:984-97, 79. doi: 10.1038/sj.mp.4001551

13. Tsai GE, Yang P, Chang YC, Chong MY. D-alanine added to antipsychotics for the treatment of schizophrenia. Biol Psychiatry. (2006) 59:2304. doi: 10.1016/j.biopsych.2005.06.032

14. Fachim HA, Loureiro CM, Corsi-Zuelli F, Shuhama R, Louzada-Junior P, Menezes PR, et al. GRIN2B promoter methylation deficits in early-onset schizophrenia and its association with cognitive function. Epigenomics. (2019) 11:401-10. doi: 10.2217/epi-2018-0127

15. Ferrarelli F, Tononi G. Reduced sleep spindle activity point to a TRN-MD thalamus-PFC circuit dysfunction in schizophrenia. Schizophr Res. (2017) 180:36-43. doi: 10.1016/j.schres.2016.05.023

16. Bartho P, Slezia A, Matyas F, Faradzs-Zade L, Ulbert I, Harris KD, et al. Ongoing network state controls the length of sleep spindles via inhibitory activity. Neuron. (2014) 82:1367-79. doi: 10.1016/j.neuron.2014. 04.046

17. Epstein KA, Cullen KR, Mueller BA, Robinson P, Lee S, Kumra S. White matter abnormalities and cognitive impairment in early-onset schizophreniaspectrum disorders. J Am Acad Child Adoles Psychiatry. (2014) 53:362-72 e1-2. doi: 10.1016/j.jaac.2013.12.007
18. Lui S, Zhou XJ, Sweeney JA, Gong Q. Psychoradiology: the frontier of neuroimaging in psychiatry. Radiology. (2016) 281:357-72. doi: 10.1148/radiol.2016152149

19. Lerch JP, van der Kouwe AJ, Raznahan A, Paus T, Johansen-Berg H, Miller KL, et al. Studying neuroanatomy using MRI. Nat Neurosci. (2017) 20:31426. doi: 10.1038/nn.4501

20. Ashburner J, Friston KJ. Voxel-based morphometry-the methods. Neuroimage. (2000) 11:805-21. doi: 10.1006/nimg.2000.0582

21. Zang YF, He Y, Zhu CZ, Cao QJ, Sui MQ, Liang M, et al. Altered baseline brain activity in children with ADHD revealed by resting-state functional MRI. Brain Dev. (2007) 29:83-91. doi: 10.1016/j.braindev.2006. 07.002

22. Tordesillas-Gutierrez D, Koutsouleris N, Roiz-Santianez R, Meisenzahl E, Ayesa-Arriola R, Marco de. Lucas E, et al. Grey matter volume differences in non-affective psychosis and the effects of age of onset on grey matter volumes: a voxelwise study. Schizophrenia research. (2015) 164:7482. doi: 10.1016/j.schres.2015.01.032

23. Tang J, Liao Y, Zhou B, Tan C, Liu W, Wang D, et al. Decrease in temporal gyrus gray matter volume in first-episode, early onset schizophrenia: an MRI study. PLoS ONE. (2012) 7:e40247. doi: 10.1371/journal.pone.0040247

24. Zheng J, Zhang Y, Guo X, Duan X, Zhang J, Zhao J, et al. Disrupted amplitude of low-frequency fluctuations in antipsychotic-naive adolescents with early-onset schizophrenia. Psychiatry Res Neuroimaging. (2016) 249:206. doi: 10.1016/j.pscychresns.2015.11.006

25. Gao ZT Li YL, Guo SQ, Xia YH. [Brain gray matter volume alterations and cognitive function in first-episode childhood-and adolescence-onset schizophrenia]. Zhonghua Yi Xue Za Zhi. (2019) 99:3581-6. doi: 10.3760/cma.j.issn.0376-2491.2019.45.010

26. Kadriu B, Gu W, Korenis P, Levine JM. Do cognitive and neuropsychological functioning deficits coincide with hippocampal alteration during first-psychotic episode? CNS Spectr. (2019) 24:472-8. doi: 10.1017/S1092852918001293

27. Shi LJ, Zhou HY, Wang Y, Shen YM, Fang YM, He YQ, et al. Altered empathyrelated resting-state functional connectivity in adolescents with early-onset schizophrenia and autism spectrum disorders. Asian J Psychiatr. (2020) 53:102167. doi: 10.1016/j.ajp.2020.102167

28. Harvey PD, Isner EC. Cognition, social cognition, and functional capacity in early-onset schizophrenia. Child Adolesc Psychiatr Clin N Am. (2020) 29:171-82. doi: 10.1016/j.chc.2019.08.008

29. Silberstein J, Harvey PD. Cognition, social cognition, and Self-assessment in schizophrenia: prediction of different elements of everyday functional outcomes. CNS Spectr. (2019) 24:88-93. doi: 10.1017/S1092852918001414

30. Bell M, Tsang HW, Greig TC, Bryson GJ. Neurocognition, social cognition, perceived social discomfort, and vocational outcomes in schizophrenia. Schizophr Bull. (2009) 35:738-47. doi: 10.1093/schbul/sbm169

31. Wexler BE, Bell MD. Cognitive remediation and vocational rehabilitation for schizophrenia. Schizophr Bull. (2005) 31:931-41. doi: 10.1093/schbul/sbi038

32. Green MF, Penn DL, Bentall R, Carpenter WT, Gaebel W, Gur RC, et al. Social cognition in schizophrenia: an NIMH workshop on definitions, assessment, and research opportunities. Schizophr Bull. (2008) 34:121120. doi: $10.1093 /$ schbul/sbm 145

33. Sergi MJ, Rassovsky Y, Widmark C, Reist C, Erhart S, Braff DL, et al. Social cognition in schizophrenia: relationships with neurocognition and negative symptoms. Schizophr Res. (2007) 90:316-24. doi: 10.1016/j.schres.2006.09.028

34. Van der Elst W, van Boxtel MP, van Breukelen GJ, Jolles J. Rey's verbal learning test: normative data for 1855 healthy participants aged 24-81 years and the influence of age, sex, education, and mode of presentation. J Int Neuropsychol Soc. (2005) 11:290-302. doi: 10.1017/S1355617705050344

35. Vasquez BP, Zakzanis KK. The neuropsychological profile of vascular cognitive impairment not demented: a meta-analysis. J Neuropsychol. (2015) 9:109-36. doi: 10.1111/jnp.12039

36. Groth-Marnat G, Baker S. Digit Span as a measure of everyday attention: a study of ecological validity. Percept Mot Skills. (2003) 97:120918. doi: 10.2466/pms.2003.97.3f.1209

37. Houx PJ, Jolles J, Vreeling FW. Stroop interference: aging effects assessed with the Stroop Color-Word Test. Exp Aging Res. (1993) 19:20924. doi: 10.1080/03610739308253934 
38. Van Rheenen TE, Rossell SL. Picture sequencing task performance indicates theory of mind deficit in bipolar disorder. J Affect Disord. (2013) 151:11324. doi: 10.1016/j.jad.2013.07.009

39. Combs DR, Penn DL, Wicher M, Waldheter E. The Ambiguous Intentions Hostility Questionnaire (AIHQ): a new measure for evaluating hostile social-cognitive biases in paranoia. Cogn Neuropsychiatry. (2007) 12:12843. doi: 10.1080/13546800600787854

40. Ashburner J, A. fast diffeomorphic image registration algorithm. Neuroimage. (2007) 38:95-113. doi: 10.1016/j.neuroimage.2007.07.007

41. Yan CG, Wang XD, Zuo XN, Zang YF, DPABI. Data processing \& analysis for (resting-state) brain imaging. Neuroinformatics. (2016) 14:33951. doi: 10.1007/s12021-016-9299-4

42. Lesh TA, Tanase C, Geib BR, Niendam TA, Yoon JH, Minzenberg MJ, et al. A multimodal analysis of antipsychotic effects on brain structure and function in first-episode schizophrenia. JAMA Psychiatry. (2015) 72:22634. doi: 10.1001/jamapsychiatry.2014.2178

43. Andreasen NC, Pressler M, Nopoulos P, Miller D, Ho BC. Antipsychotic dose equivalents and dose-years: a standardized method for comparing exposure to different drugs. Biol Psychiatry. (2010) 67:255-62. doi: 10.1016/j.biopsych.2009.08.040

44. Liao J, Yan H, Liu Q, Yan J, Zhang L, Jiang S, et al. Reduced paralimbic system gray matter volume in schizophrenia: correlations with clinical variables, symptomatology and cognitive function. J Psychiatr Res. (2015) 65:80-6. doi: 10.1016/j.jpsychires.2015.04.008

45. Jiang Y, Duan M, Chen X, Zhang X, Gong J, Dong D, et al. Aberrant prefrontal-thalamic-cerebellar circuit in schizophrenia and depression: evidence from a possible causal connectivity. Int J Neural Syst. (2019) 29:1850032. doi: 10.1142/S0129065718500326

46. Zhang YY, Liao JM, Li QQ, Zhang X, Liu LJ, Yan J, et al. Altered restingstate brain activity in schizophrenia and obsessive-compulsive disorder compared with non-psychiatric controls: commonalities and distinctions across disorders. Front Psychiatry. (2021) 12:681701. doi: 10.3389/fpsyt.2021. 681701

47. Li Z, Lei W, Deng W, Zheng Z, Li M, Ma X, et al. Aberrant spontaneous neural activity and correlation with evoked-brain potentials in first-episode, treatment-naive patients with deficit and non-deficit schizophrenia. Psychiatry Res Neuroimaging. (2017) 261:9-19. doi: 10.1016/j.pscychresns.2017. 01.001

48. Li Q, Cao X, Liu S, Li Z, Wang Y, Cheng L, et al. Dynamic alterations of amplitude of low-frequency fluctuations in patients with drug-naive first-episode early onset schizophrenia. Front Neurosci. (2020) 14:901. doi: 10.3389/fnins.2020.00901

49. Kapur N, Ellison D, Smith MP, McLellan DL, Burrows EH. Focal retrograde amnesia following bilateral temporal lobe pathology. A neuropsychological and magnetic resonance study. Brain. (1992) 115(Pt. 1):73-85. doi: 10.1093/brain/115.1.73

50. Maguire EA, Mummery CJ. Differential modulation of a common memory retrieval network revealed by positron emission tomography. Hippocampus. (1999) 9:54-61.

51. Tomadesso C, Perrotin A, Mutlu J, Mezenge F, Landeau B, Egret S, et al. Brain structural, functional, and cognitive correlates of recent versus remote autobiographical memories in amnestic Mild Cognitive Impairment. Neuroimage Clin. (2015) 8:473-82. doi: 10.1016/j.nicl.2015.05.010

52. Marinkovic K, Dhond RP, Dale AM, Glessner M, Carr V, Halgren E. Spatiotemporal dynamics of modality-specific and supramodal word processing. Neuron. (2003) 38:487-97. doi: 10.1016/S0896-6273(03) 00197-1

53. Mesulam MM, Rogalski EJ, Wieneke C, Hurley RS, Geula C, Bigio EH, et al. Primary progressive aphasia and the evolving neurology of the language network. Nat Rev Neurol. (2014) 10:554-69. doi: 10.1038/nrneurol. 2014.159

54. Chadwick MJ, Anjum RS, Kumaran D, Schacter DL, Spiers HJ, Hassabis D. Semantic representations in the temporal pole predict false memories. Proc Natl Acad Sci USA. (2016) 113:10180-5. doi: 10.1073/pnas. 1610686113

55. Jimura K, Konishi S, Miyashita Y. Temporal pole activity during perception of sad faces, but not happy faces, correlates with neuroticism trait. Neurosci Lett. (2009) 453:45-8. doi: 10.1016/j.neulet.2009.02.012
56. Frith U, Frith CD. Development and neurophysiology of mentalizing. Philosophical transactions of the Royal Society of London Series B, Biological sciences. (2003) 358:459-73. doi: 10.1098/rstb.2002.1218

57. Pehrs C, Zaki J, Schlochtermeier LH, Jacobs AM, Kuchinke L, Koelsch S. The temporal pole top-down modulates the ventral visual stream during social cognition. Cerebral Cortex. (2017) 27:777-92. doi: 10.1093/cercor/ bhv226

58. McClelland JL, Rogers TT. The parallel distributed processing approach to semantic cognition. Nat Rev Neurosci. (2003) 4:310-22. doi: 10.1038/nrn1076

59. Patterson K, Nestor PJ, Rogers TT. Where do you know what you know? The representation of semantic knowledge in the human brain. Nat Rev Neurosci. (2007) 8:976-87. doi: 10.1038/nrn2277

60. Joubert S, Gour N, Guedj E, Didic M, Gueriot C, Koric L, et al. Early-onset and late-onset Alzheimer's disease are associated with distinct patterns of memory impairment. Cortex. (2016) 74:217-32. doi: 10.1016/j.cortex.2015.10.014

61. Campo P, Poch C, Toledano R, Igoa JM, Belinchon M, Garcia-Morales I, et al. Visual object naming in patients with small lesions centered at the left temporopolar region. Brain Struct Funct. (2016) 221:47385. doi: 10.1007/s00429-014-0919-1

62. Petrides M. Lateral prefrontal cortex: architectonic and functional organization. Philos Trans $R$ Soc Lond Ser B Biol Sci. (2005) 360:781-95. doi: 10.1098/rstb.2005.1631

63. Muller NG, Knight RT. The functional neuroanatomy of working memory: contributions of human brain lesion studies. Neuroscience. (2006) 139:518. doi: 10.1016/j.neuroscience.2005.09.018

64. Koechlin E, Summerfield C. An information theoretical approach to prefrontal executive function. Trends Cogn Sci. (2007) 11:229-35. doi: 10.1016/j.tics.2007.04.005

65. Bortolato B, Miskowiak KW, Kohler CA, Vieta E, Carvalho AF. Cognitive dysfunction in bipolar disorder and schizophrenia: a systematic review of meta-analyses. Neuropsychiatr Dis Treat. (2015) 11:3111-25. doi: 10.2147/NDT.S76700

66. Poppe AB, Barch DM, Carter CS, Gold JM, Ragland JD, Silverstein SM, et al. Reduced frontoparietal activity in schizophrenia is linked to a specific deficit in goal maintenance: a multisite functional imaging study. Schizophr Bull. (2016) 42:1149-57. doi: 10.1093/schbul/sbw036

67. Rizzolatti G, Craighero L. The mirror-neuron system. Annu Rev Neurosci. (2004) 27:169-92. doi: 10.1146/annurev.neuro.27.070203.144230

68. Jani M, Kasparek T. Emotion recognition and theory of mind in schizophrenia: a meta-analysis of neuroimaging studies. World J Biol Psychiatry. (2018) 19:S86-96. doi: 10.1080/15622975.2017.1324176

69. Cook R, Bird G, Catmur C, Press C, Heyes C. Mirror neurons: from origin to function. Behav Brain Sci. (2014) 37:17792. doi: 10.1017/S0140525X13000903

70. Sun $F$, Zhao Z, Lan $M, X u$ Y, Huang $M, X u ~ D$. Abnormal dynamic functional network connectivity of the mirror neuron system network and the mentalizing network in patients with adolescentonset, first-episode, drug-naive schizophrenia. Neurosci Res. (2021) 162:63-70. doi: 10.1016/j.neures.2020.01.003

71. Choe E, Lee TY, Kim M, Hur JW, Yoon YB, Cho KK, et al. Aberrant withinand between-network connectivity of the mirror neuron system network and the mentalizing network in first episode psychosis. Schizophr Res. (2018) 199:243-9. doi: 10.1016/j.schres.2018.03.024

72. Xu J, Lyu H, Li T, Xu Z, Fu X, Jia F, et al. Delineating functional segregations of the human middle temporal gyrus with resting-state functional connectivity and coactivation patterns. Hum Brain Mapp. (2019) 40:5159-71. doi: 10.1002/hbm.24763

73. Schurz M, Radua J, Aichhorn M, Richlan F, Perner J. Fractionating theory of mind: a meta-analysis of functional brain imaging studies. Neurosci Biobehav Rev. (2014) 42:9-34. doi: 10.1016/j.neubiorev.2014.01.009

74. Schurz M, Tholen MG, Perner J, Mars RB, Sallet J. Specifying the brain anatomy underlying temporo-parietal junction activations for theory of mind: a review using probabilistic atlases from different imaging modalities. Hum Brain Mapp. (2017) 38:4788-805. doi: 10.1002/hbm.23675

75. Lee JS, Kim CY, Joo YH, Newell D, Bouix S, Shenton ME, et al. Increased diffusivity in gray matter in recent onset schizophrenia is associated with clinical symptoms and social cognition. Schizophr Res. (2016) 176:14450. doi: $10.1016 /$ j.schres.2016.08.011 
Conflict of Interest: The authors declare that the research was conducted in the absence of any commercial or financial relationships that could be construed as a potential conflict of interest.

The Handling Editor YT declared a shared affiliation, though no other collaboration, with one of the authors PG, SH, XJ, HoZ, DM, XC, JZ, and HuZ at the time of the review.

Publisher's Note: All claims expressed in this article are solely those of the authors and do not necessarily represent those of their affiliated organizations, or those of the publisher, the editors and the reviewers. Any product that may be evaluated in this article, or claim that may be made by its manufacturer, is not guaranteed or endorsed by the publisher.

Copyright (c) 2022 Guo, Hu, Jiang, Zheng, Mo, Cao, Zhu and Zhong. This is an open-access article distributed under the terms of the Creative Commons Attribution License (CC BY). The use, distribution or reproduction in other forums is permitted, provided the original author(s) and the copyright owner(s) are credited and that the original publication in this journal is cited, in accordance with accepted academic practice. No use, distribution or reproduction is permitted which does not comply with these terms. 